\title{
Efficiency-limiting processes in cyclopentadithiophene-bridged donor-acceptor-type dyes for solid-state dye-sensitized solar cells
}

Felix Hinkel, Yoojin M. Kim, Yulian Zagraniarsky, Florian Schlütter, Denis Andrienko, Klaus Müllen, and Frédéric Laquai

Citation: The Journal of Chemical Physics 148, 044703 (2018);

View online: https://doi.org/10.1063/1.4999136

View Table of Contents: http://aip.scitation.org/toc/jcp/148/4

Published by the American Institute of Physics

\section{Articles you may be interested in}

Communication: Modeling electrolyte mixtures with concentration dependent dielectric permittivity

The Journal of Chemical Physics 148, 041102 (2018); 10.1063/1.5018195

Combining the ensemble and Franck-Condon approaches for calculating spectral shapes of molecules in solution

The Journal of Chemical Physics 148, 024110 (2018); 10.1063/1.5006043

Simulating structure and dynamics in small droplets of 1-ethyl-3-methylimidazolium acetate

The Journal of Chemical Physics 148, 193802 (2018); 10.1063/1.5010342

\section{AIP $\left.\right|^{\text {The Joumal of }}$ Chemical Physics}




\title{
Efficiency-limiting processes in cyclopentadithiophene-bridged donor-acceptor-type dyes for solid-state dye-sensitized solar cells
}

\author{
Felix Hinkel, ${ }^{1, a)}$ Yoojin M. Kim, ${ }^{1, a)}$ Yulian Zagraniarsky, ${ }^{1}$ Florian Schlütter, ${ }^{1}$ \\ Denis Andrienko, ${ }^{1}$ Klaus Müllen, ${ }^{1, b)}$ and Frédéric Laquai ${ }^{2, c)}$ \\ ${ }^{1}$ Max-Planck-Institute for Polymer Research, Ackermannweg 10, D-55128 Mainz, Germany \\ ${ }^{2}$ King Abdullah University of Science and Technology (KAUST), KAUST Solar Center (KSC), \\ Physical Sciences and Engineering Division (PSE), Material Science and Engineering Program (MSE), \\ Thuwal 23955-6900, Kingdom of Saudi Arabia
}

(Received 5 August 2017; accepted 8 January 2018; published online 26 January 2018)

\begin{abstract}
The charge generation and recombination processes in three novel push-pull photosensitizers for dyesensitized solar cells (DSSCs) are studied by ps- $\mu$ s transient absorption (TA) and quasi-steady-state photoinduced absorption (PIA) spectroscopy. The three cyclopentadithiophene-based photosensitizer dye molecules exhibit comparably low power conversion efficiencies ranging from $0.8 \%$ to $1.7 \%$ in solid-state DSSCs. We find that the photocurrents increase in the presence of Li-salt additives. Both TA and PIA measurements observe long-lived dye cations created by electron injection from the dyes' excited state for two dyes from the series. However, the third dye shows significantly lower performance as a consequence of the less efficient electron injection even after the addition of Li-salts and faster electron-hole recombination on the ns- $\mu$ s time scale. In essence, the prerequisites for this class of donor- $\pi$ bridge-acceptor photosensitizers to reach higher charge generation efficiencies are a combination of strong dipole moments and fine tuning of the electronic landscape at the titania-dye interface by Li-salt addition. Published by AIP Publishing. https://doi.org/10.1063/1.4999136
\end{abstract}

\section{INTRODUCTION}

The concept of dye-sensitized solar cells (DSSCs) has attracted considerable attention due to the potential for lowcost production of non-toxic solar cells with high power conversion efficiencies (PCE) since it was originally introduced in $1991 .{ }^{1}$ Owing to a better understanding of the device photophysics and the concurrent development of novel materials including dyes, redox mediators, hole transporters, and additives, DSSCs have reached efficiencies now exceeding $12 \%$ in liquid-electrolyte devices, while the efficiency remained for long at $7 \%$ for the best solid-state devices. ${ }^{2-4}$ In liquidelectrolyte DSSCs, higher PCEs can be achieved due to slower charge recombination kinetics as well as long excitedstate lifetimes, especially if organometallic complexes such as ruthenium $(\mathrm{Ru}(\mathrm{II}))$ dye complexes are used. ${ }^{5-8}$ However, all-organic dyes are being developed as alternatives, as they exhibit several promising advantages such as high molecular extinction coefficients $\left(>20000 \mathrm{~L} \mathrm{~mol}^{-1} \mathrm{~cm}^{-1}\right.$ ), tunable absorption bands, facile synthesis, and molecular design flexibility in combination with lower production costs due to the exclusion of expensive second and third row transition metals. All-organic sensitizers often employ a donor- $\pi$ bridge-acceptor (D- $\pi-\mathrm{A})$ motif to separate the highest occupied molecular orbital (HOMO) from the lowest unoccupied molecular orbital (LUMO), of which the latter is located close to the dyes' anchor group to facilitate electron injection into

\footnotetext{
a)F. Hinkel and Y. M. Kim contributed equally to this work.

b)muellen@mpip-mainz.mpg.de

c)frederic.laquai@kaust.edu.sa
}

titania. ${ }^{9,10}$ Among many organic photosensitizers, devices that use cobalt (II/III) complexes as a hole conductor have shown excellent efficiencies when, especially, electron-rich triphenylamine (TPA) moieties and thiophene derivatives such as cyclopentadithiophene (CDT) were used as electron donors and $\pi$-conjugated spacers, respectively. ${ }^{1-16}$ High-efficiency CDT-TPA dye molecules have previously shown PCEs up to $12 \%$ in liquid-state DSSCs and a solid-state DSSC employing spiro-OMeTAD (2,2'7,7'-tetrakis-( $N, N$-di- $p$-methoxyphenylamine)-9, $9^{\prime}$-spirobifluorene) showed an efficiency of $9 \%$ using a CDT derivative dye, namely, Y123, as a photosensitizer. ${ }^{17}$ The success of this dye is believed to be due to strong intramolecular charge transfer (ICT) characteristics resulting from a combination of the electron-donating TPA, the conjugated spacer CDT, and the electron-withdrawing anchor group.

In this work, we present three structurally related D- $\pi$ A photosensitizers (see Fig. 1 for chemical structures), which use CDT and TPA-based building blocks as bridging and donor units, respectively. The TPA donor-units used here were chosen, as they exhibit certain structural attributes, which are expected to have an impact on the photophysics and device performance: indolo[3,2,1-jk]carbazole (1) provides extended $\pi$-conjugation and planarity, the triangulene type triarylamine (2) shows reduced aggregation through steric hindrance of the methylene groups, and the dendritic structure (3) is a strong donor-unit with a half wave potential $\left(\mathrm{E}_{1 / 2}\right)$ of $+0.55 \mathrm{~V} \cdot{ }^{18}$ Steady-state spectroscopy techniques were used to study the optical properties of these dyes, and broadband transient absorption spectroscopy (TAS) was employed to investigate the excited-state dynamics of transient states such as excitons, 


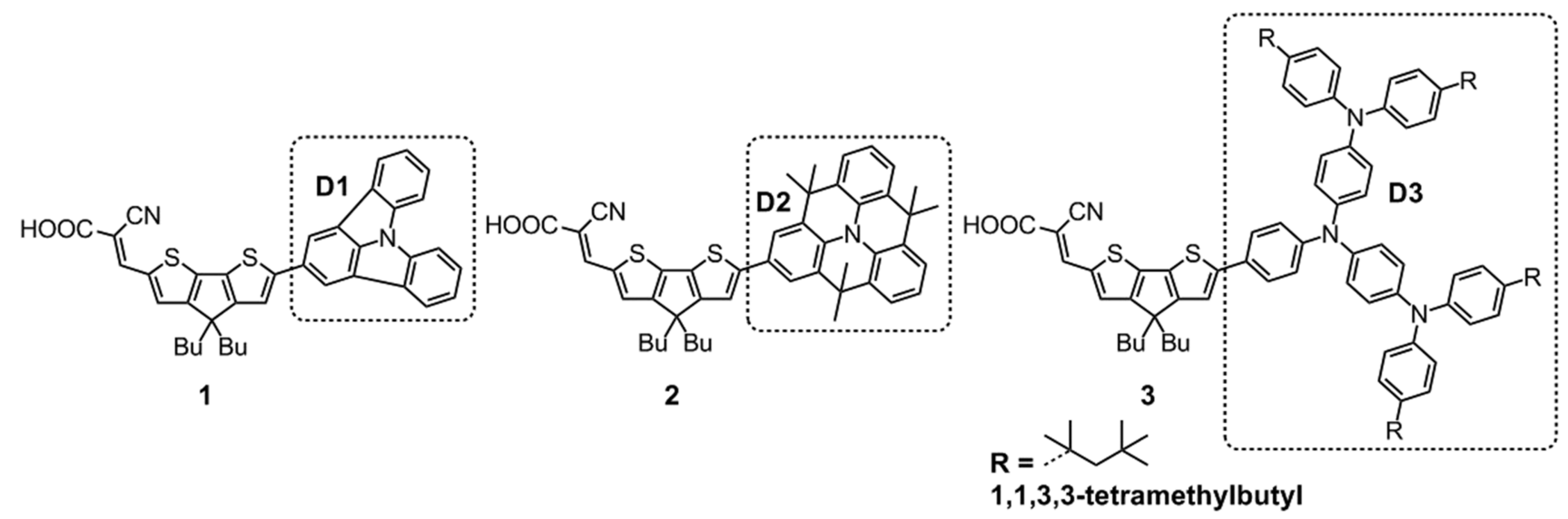

FIG. 1. Molecular structures of dyes 1-3. The donor-units are highlighted; D1 indolo[3,2,1-jk]carbazole, D2 triangulene-type TPA, and D3 dendritic TPA.

charge-transfer states, and charges in device-like samples. The combination of well-selected dye structures and advanced spectroscopy techniques allows us to understand the efficiency-limiting processes in this class of dyes and derive structure-property relations for a better-guided material design.

\section{RESULTS AND DISCUSSION}

The steady-state optical properties of dyes $\mathbf{1 - 3}$ were investigated by UV-vis absorption and photoluminescence (PL) spectroscopy, and the electrochemical characteristics by cyclic voltammetry (Table I) using an $\mathrm{Ag} / \mathrm{AgCl}$ reference electrode. UV-vis absorption spectra showed pronounced maxima in toluene at $424 \mathrm{~nm}(\mathbf{1}), 412 \mathrm{~nm}(\mathbf{2})$, and $507 \mathrm{~nm}(\mathbf{3})$ and in THF at $465 \mathrm{~nm}(\mathbf{1}), 480 \mathrm{~nm}(\mathbf{2})$, and $503 \mathrm{~nm} \mathrm{(3).} \mathrm{In} \mathrm{toluene,} \mathbf{1}$ and 2 showed maxima at around $420 \mathrm{~nm}$ with a less-pronounced bathochromic shoulder at around $450-550 \mathrm{~nm}$. Increasing the polarity of the solvent, the positive solvatochromism for $\mathbf{1}$ and 2 was seen in a bathochromic shift in light absorption. On the other hand, the absorption maximum mainly remained unchanged for $\mathbf{3}$ in either solvent. The maximal molar extinction coefficients $(\varepsilon)$ in THF were found to be $6 \times 10^{4} \mathrm{~L} \mathrm{~mol}^{-1}$ $\mathrm{cm}^{-1}$ for $\mathbf{1}$ and $\mathbf{2}$, and the value for $\mathbf{3}$ was found to be $4 \times 10^{4} \mathrm{~L} \mathrm{~mol}^{-1} \mathrm{~cm}^{-1}$. The molar extinction coefficient of 2 is in line with that of a photosensitizer with similar molecular structure, namely, LC-3, reported previously. ${ }^{19}$ We note that the molar extinction coefficients of these photosensitizers are three times higher than that of the Ru(II) complex dye Z907 $\left(1.3 \times 10^{4} \mathrm{~L} \mathrm{~mol}^{-1} \mathrm{~cm}^{-1}\right.$ at $\left.520 \mathrm{~nm}\right)$, which was used in the present study as a reference dye. Thus, the CDT-TPA series of dye molecules could potentially exhibit high photocur-

TABLE I. Data from UV-vis and PL spectroscopy (in THF solution, $10^{-5} \mathrm{M}$ ) and cyclic voltammetry. ${ }^{20}$

\begin{tabular}{lccccc}
\hline \hline $\begin{array}{l}\text { Dye } \\
\text { molecule }\end{array}$ & $\begin{array}{c}\lambda_{\max } \\
(\mathrm{nm})\end{array}$ & $\begin{array}{c}\lambda_{\text {onset }} \\
(\mathrm{nm})\end{array}$ & $\begin{array}{c}\Delta \mathrm{E}_{\mathrm{Gap}} \\
(\mathrm{eV})\end{array}$ & $\begin{array}{c}\lambda_{\mathrm{FL}, \max } \\
(\mathrm{nm})\end{array}$ & $\begin{array}{c}\mathrm{E}_{\mathrm{HOMO}} \\
(\mathrm{eV})\end{array}$ \\
\hline 1 & 465 & 503 & 2.66 & 672 & -5.95 \\
2 & 480 & 600 & 2.58 & 663 & -5.06 \\
3 & 503 & 636 & 2.46 & 660 & -5.10 \\
\hline \hline
\end{tabular}

rents due to their high molar extinction coefficients. Density functional theory (DFT) calculations using the b3lyp/6$311 \mathrm{~g}(\mathrm{~d}, \mathrm{p})$ functional (see the supplementary material) indicate that the HOMO of the dyes is delocalized across the entire molecules, while the LUMO is localized on the acceptor/ anchor group.

The redoxpotentials of these three dye molecules were measured by cyclic voltammetry (CV) using $0.1 \mathrm{M}$ dichloromethane solution with an $\mathrm{Ag} / \mathrm{AgCl}$ reference electrode and a platinum counter electrode giving reversible oxidation peaks (see the supplementary material) and used to estimate the HOMO energies by using ferrocene as reference. The HOMO energies of this series of dyes are below the HOMO energy of spiro-OMeTAD $(-5.95 \mathrm{eV}$ for $\mathbf{1},-5.06 \mathrm{eV}$ for 2 , and $-5.10 \mathrm{eV}$ for $\mathbf{3}$ ), in turn allowing efficient hole transfer (dye cation reduction) and thus dye regeneration. DFT calculations (see the supplementary material, Table S1) confirm this trend and indicate that dye $\mathbf{1}$ has the highest ionization potential (IP) in this series of dyes. The LUMO energy could not be deduced from $\mathrm{CV}$ measurements, as no reduction wave was observed. Thus, the LUMO levels were derived from the onsets of absorption of dyes in THF solutions, yielding $-2.28 \mathrm{eV}$ for $\mathbf{1},-2.24 \mathrm{eV}$ for $\mathbf{2}$, and $-2.45 \mathrm{eV}$ for $\mathbf{3}$. The LUMO levels of the three sensitizers allow electron injection into the conduction band of titania after photoexcitation of the dye molecules. However, the HOMO levels may not be suitable for a liquid-state DSSC using cobalt salts as electrolytes, which have reduction potentials corresponding to -5.2 to $-5.4 \mathrm{eV}$.

Figure 2 depicts the J-V curves of solid-state DSSCs that use 1 (black), $\mathbf{2}$ (blue), $\mathbf{3}$ (red), and the commercial dye Z907 (black dashed) as photosensitizers measured under solar-like illumination of AM1.5G. 2 gave the highest efficiency of $1.7 \%$ followed by 1 with $1.6 \% .1$ and 2 exhibited higher shortcircuit currents than the photocurrent of the reference device using the Ru-dye Z907. The Z907 device revealed a PCE of $1.8 \%$ with a short-circuit current of $3.31 \mathrm{~mA} / \mathrm{cm}^{2}$, an opencircuit voltage of $0.86 \mathrm{~V}$, and a fill factor of $63 \%$. The inset of Fig. 2 shows the internal quantum efficiencies (IQE) of the three different devices. The three sensitized-films absorb in the wavelength range between 400 and $600 \mathrm{~nm}$ where a large number of photons are present in the spectrum of the sun. The IQEs 


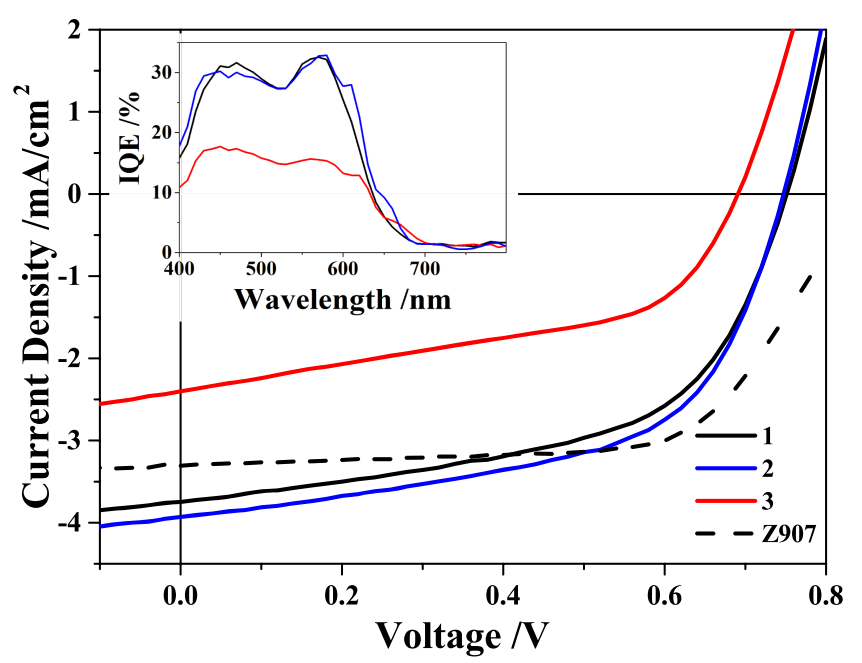

FIG. 2. J-V curves of solid-state DSSC using 1 (black), 2 (blue), 3 (red), and Z907 (black dashed) measured under $100 \mathrm{~mW} / \mathrm{cm}^{2}$ AM $1.5 \mathrm{G}$ solar illumination. Inset: internal quantum efficiency (IQE) of each device.

of both $\mathbf{1}$ and $\mathbf{2}$ were rather constant at around 30\% throughout the absorption range, whereas 3 could reach only $17 \%$. From the sensitized films' absorption spectra, the maximum obtainable $\mathrm{J}_{\mathrm{SC}}$ was calculated by assuming $100 \% \mathrm{IQE}$. The maximum short-circuit currents that could be reached are $12.6 \mathrm{~mA} / \mathrm{cm}^{2}(\mathbf{1}), 13.5 \mathrm{~mA} / \mathrm{cm}^{2}(\mathbf{2})$, and $12.2 \mathrm{~mA} / \mathrm{cm}^{2}(\mathbf{3})$, for an IQE of unity. This indicates that only $30 \%, 29 \%$, and $20 \%$ of the expected short-circuit currents were experimentally achieved in devices that use $\mathbf{1}, \mathbf{2}$, and $\mathbf{3}$ as sensitizers, respectively, which agrees with the values of the IQEs as shown above.

Time-resolved PL spectroscopy on dye molecules adsorbed on different metal oxide nanoparticles, namely, $\mathrm{Al}_{2} \mathrm{O}_{3}$ and $\mathrm{TiO}_{2}$, was performed. These two metal oxides were chosen as the high conduction band of alumina allows studying the excited state dynamics of the dyes attached to a metal oxide surface, but in the absence of electron injection, while on titania electron injection can occur. The PL quenching efficiency was determined by comparing the PL signal intensities of the samples keeping the measurement conditions the same across the series of measurements. Figure 3(a) shows the PL dynamics of dyes adsorbed on $\mathrm{Al}_{2} \mathrm{O}_{3}$ particles (solid lines) in comparison to those adsorbed on $\mathrm{TiO}_{2}$ surface (dashed lines). This comparison provides an estimate of how efficiently the dyes inject electrons into $\mathrm{TiO}_{2}$. Interestingly, 3 showed the highest injection efficiency (IE) among the dye molecules of about $97 \%$, whereas $\mathbf{1}$ and $\mathbf{2}$ exhibited $81 \%$ and 63\%, respectively. Further details of this calculation can be found in the supplementary material.

On a $\mathrm{TiO}_{2}$ surface, electron injection occurs and we observe PL from the fraction of dye molecules that do not take part in the injection process. In fact, electron injection in DSSCs usually occurs on a femto- to pico-second time scale on titania and thus reduces the PL signal amplitude observed in our experiments. Upon exposure to Li-ions, the PL intensity and dynamics change and the dye molecules reveal some interesting details of the injection process, presented in panel (b). Here, we observe two pools of dye molecules: one pool is affected by the addition of Li-ions, while Li-ions do not affect
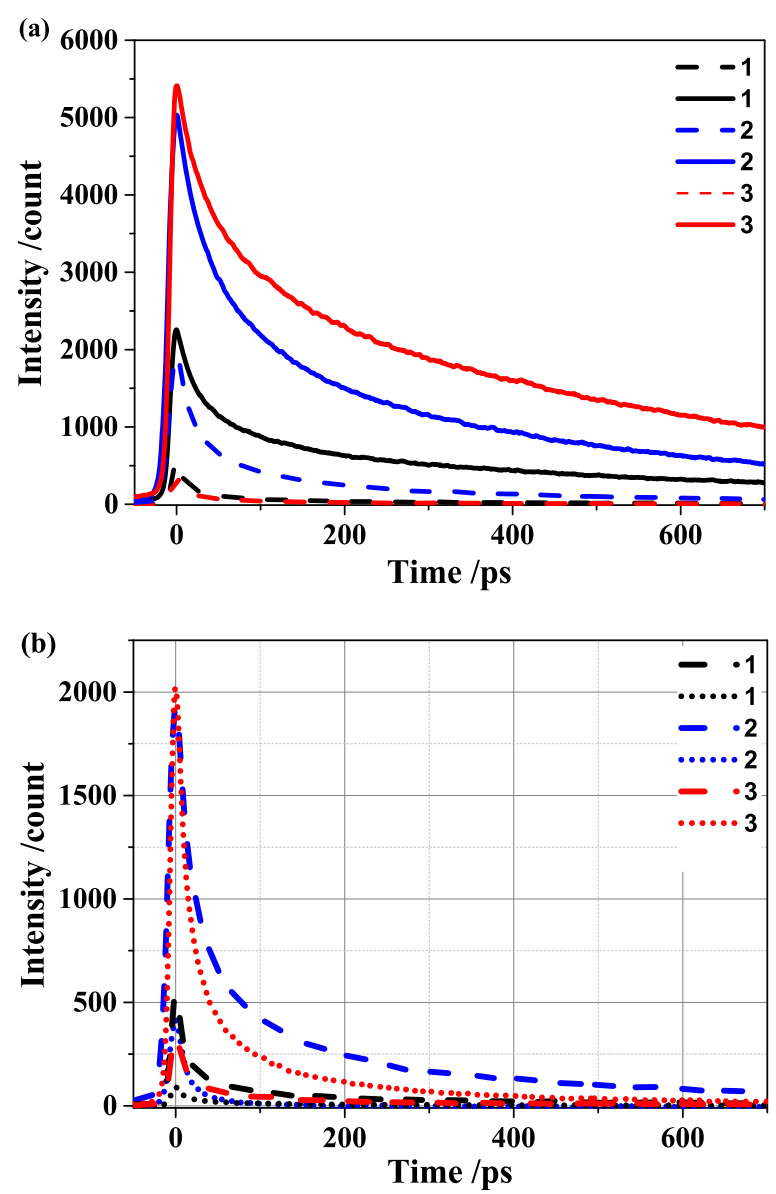

FIG. 3. Photoluminescence decay curves of (a) $\mathbf{1}$ (black), $\mathbf{2}$ (blue), and $\mathbf{3}$ (red) on $\mathrm{Al}_{2} \mathrm{O}_{3}$ (solid lines) and on $\mathrm{TiO}_{2}$ (dashed lines), and of (b) dye molecules on $\mathrm{TiO}_{2}$ in the absence (dashed lines) and presence (dotted lines) of Li cations.

the other pool. In fact, addition of Li-ions might activate the injection process of some dye molecules that previously could not contribute to charge generation. A similar concept has previously been used to explain increased IEs on nanostructured $\mathrm{TiO}_{2}$ surfaces after application of Li salts. ${ }^{21}$

After application of Li-salts, 2 exhibited the highest IE of $95 \%$, followed by $\mathbf{1}$ with an IE of $84 \%$. Interestingly, when the films were treated with Li-ions, some of the PL was recovered in the case of dye $\mathbf{3}$ on $\mathrm{TiO}_{2}$ (IE of 65\%). The overall IE on Li-ion-treated- $\mathrm{TiO}_{2}$ surfaces in comparison to $\mathrm{Al}_{2} \mathrm{O}_{3}$ surfaces is $99 \%$ for $\mathbf{1}$ and $\mathbf{2}$, that is, close to unity, whilst 3 resulted in an IE of $87 \% . \mathbf{1}$ and $\mathbf{2}$ appear to be more affected by the presence of Li-ions, as electron injection into $\mathrm{TiO}_{2}$ nanoparticles occurred more efficiently in their presence; however, it appears that some of the excited-states of $\mathbf{3}$ did not inject after the addition of Li-ions. This is supported by TA measurements (see Fig. S6.1, panel (c) in the supplementary material), showing a decreased amplitude of the TA signal in the NIR spectral range and similar decay kinetics to those observed in samples without Li-additives, indicating that $\mathrm{Li}$-ions cannot aid the charge generation in this case. Hence, devices using dyes $\mathbf{1}$ and $\mathbf{2}$ should achieve higher photocurrents than devices using $\mathbf{3}$, which is also observed experimentally.

Figure 4 compares the absorption spectra of the dyes in solution using toluene (TLN) and tetrahydrofuran (THF) 


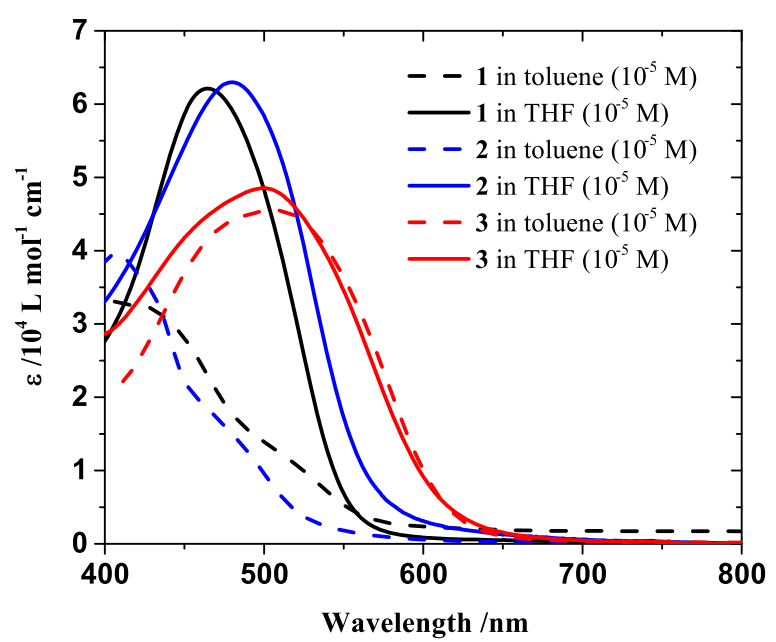

FIG. 4. UV-vis spectra of $\mathbf{1}$ (black), $\mathbf{2}$ (blue), and $\mathbf{3}$ (red) at a concentration of $0.1 \mathrm{mM}$ in toluene (dotted) and tetrahydrofuran (THF, solid).

as solvents (vide supra). We note that a bathochromic shift of the absorption with increasing solvent polarity was found for $\mathbf{1}$ and $2 .{ }^{22}$ As we demonstrate below, this allows us to deduce the presence of a local electric field causing a shift of the absorption on sensitized $\mathrm{TiO}_{2}$ films when Li-ions are used as additive. Li-ions cannot only reside within or on the surface of $\mathrm{TiO}_{2}$ nanoparticles and thereby create a polar environment next to the dye molecules at the interface, but also lower the conduction band of titania, so that charge injection from the photosensitizer to the nanoparticles becomes energetically more favorable. In fact, the large Stokes shifts of $\mathbf{1}$ and 2 observed in the presence of Li-ions indicate that these dye molecules have a larger dipole moment in the excited-state than in the ground-state, leading to a more favorable electron injection process. ${ }^{22-24}$ (See Fig. S8 in the supplementary material.) Interestingly, the absorption and emission spectra of 3 blue-shifted upon addition of Li cations. Absorption measurements with solvents of increasing polarity confirmed this observation, as they showed a weak negative solvatochromism for 3. Presumably, this leads to less favorable electron injection properties in DSSCs, when Li-ions are added, since they similarly create a polar environment in the vicinity of dye molecules.

Apart from the IE calculation, further rationale for the higher photocurrents of $\mathbf{1}$ and $\mathbf{2}$ can be found from quasisteady-state photoinduced absorption (PIA) measurements, which monitor the presence of long-lived excited states, here: charges. Figure 5 shows the PIA spectra obtained from different samples: dyes on $\mathrm{TiO}_{2}$ [panel (a)], dyes on $\mathrm{TiO}_{2}$ after addition of Li-salts [panel (b)], and in a solar cell-like structure using $\mathrm{TiO}_{2}\left(\mathrm{TiO}_{2} /\right.$ dye/spiro-OMeTAD $\left.+\mathrm{Li}-\mathrm{TFSI}+t \mathrm{BP}\right)$ [panel (c)]. Among the series of photosensitizers, 1 (black) revealed the strongest ground-state bleaching (GSB) and a Stark effect up to $600 \mathrm{~nm}$ as well as photoinduced absorption (PA) signals between 600 and $1600 \mathrm{~nm}$, indicating that charges were generated efficiently, and that they had a long lifetime even in the absence of any $\mathrm{Li}$ additive, as shown in panel (a). Dye 2 (blue) exhibited the lowest signal amplitude, implying only a small amount of electron injection and/or a short charge carrier lifetime. Dye $\mathbf{3}$ (red) showed negative PIA

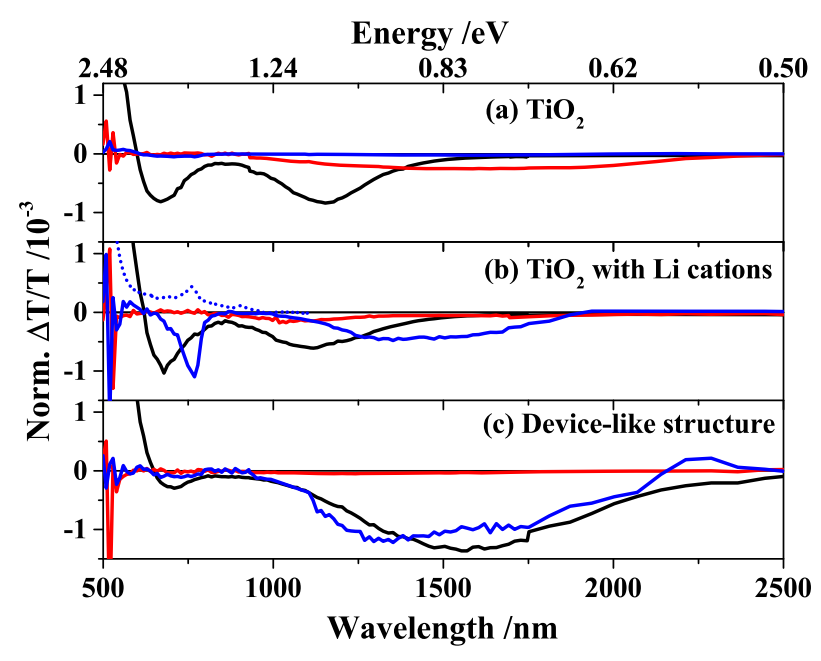

FIG. 5. Quasi-steady-state photoinduced absorption spectra of $\mathbf{1}$ (black), 2 (blue), and $\mathbf{3}$ (red) adsorbed on $\mathrm{TiO}_{2}$ (a), of the three dye molecules on $\mathrm{TiO}_{2}$ after application of Li cations (b), and in device-like structures (c).

signals in the NIR $(800 \mathrm{~nm}-2500 \mathrm{~nm})$, but almost no PA signal in the visible range, indicating poor charge generation and/or faster charge recombination.

In panel (b), $\mathbf{1}$ and $\mathbf{2}$ are seen to exhibit strong PIA signals ranging from $600 \mathrm{~nm}$ to $1600 / 1900 \mathrm{~nm}$, which were assigned to the absorption of dye cations created by electron injection. 2 displayed a very sharp peak around $770 \mathrm{~nm}$ that originates from the absorption of its cation (dotted blue line). The absorption of the cations as a result of the electron injection process was confirmed by steady-state absorption measurements on dye solutions in the presence of iron(III)chloride $\left(\mathrm{FeCl}_{3}\right)$, where $\mathrm{FeCl}_{3}$ acts as a Lewis acid and oxidizes the dyes. For 3, the amplitude of the signal was about 4 times smaller than that observed for $\mathbf{1}$ as shown in panel (a) and the PIA signal was blue-shifted to $700-1600 \mathrm{~nm}$ from 800 to $2400 \mathrm{~nm}$. This is in line with the results of transient PL measurements as presented in Fig. 3(b), which demonstrated higher IE (more PL quenching) in the absence of Li-ions.

Panel (c) shows the PIA spectra of complete devicelike samples. The steady-state absorption spectrum of spiroOMeTAD cations can be used as a measure of the dye cation regeneration process. $\mathbf{1}$ and $\mathbf{2}$ gave rise to strong absorption signals around $1500 \mathrm{~nm}$ where the oxidized hole conductor, namely, spiro-OMeTAD, mostly absorbs, whereas 3 showed almost no absorption of the oxidized spiro-OMeTAD in the near-infrared region, in good agreement with the poor efficiency observed in devices that use this dye. However, we note that the signal amplitude in PIA experiments cannot be used as a direct measure of the charge generation yield, since the PIA signal intensity is a convolution of the carrier concentration and lifetime.

Further investigation of the electron injection, charge generation, and recombination processes on a picosecond to nanosecond time scale was performed with TAS since PIA can only monitor the presence of long-lived charges, present on a nano- to micro-second time scale. Figures 6(a), 6(c), and 6(e) compare the transient absorption spectra of $\mathbf{1}, \mathbf{2}$, and $\mathbf{3}$ on titania after treatment with Li-ions, and panel (b), (d), and (f) depict those of the complete device-like structures (without 


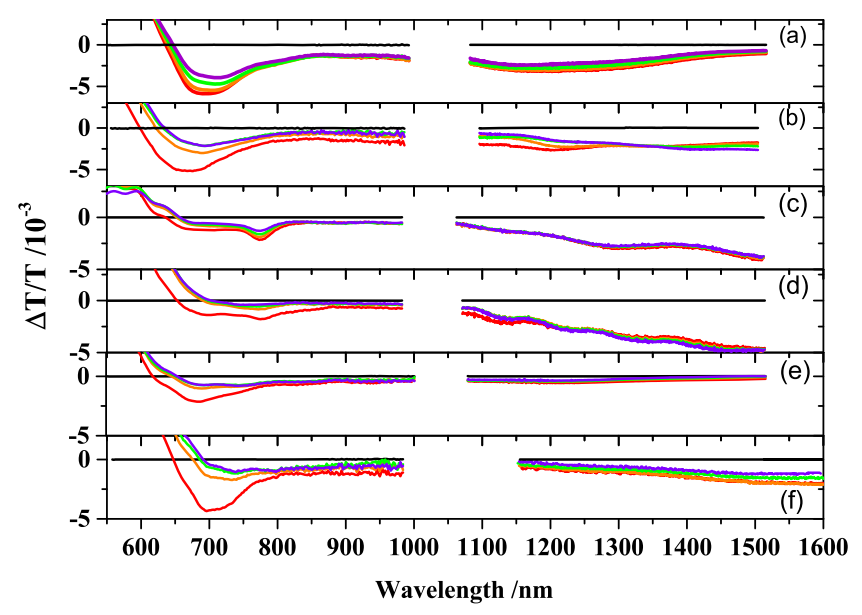

FIG. 6. Short delay (ps-ns) broadband TA spectra of $\mathbf{1}$ (a), 2 (c), and $\mathbf{3}$ (e) on $\mathrm{TiO}_{2}$ after addition of Li-ions and of $\mathbf{1}$ (b), $\mathbf{2}$ (d), and $\mathbf{3}$ (f) on $\mathrm{TiO}_{2}$ with LiTFSI/tBP/spiro-OMeTAD (complete device structure) at delay times of $3 \mathrm{ps}$ (red), $30 \mathrm{ps}$ (orange), $300 \mathrm{ps}$ (green), and $3 \mathrm{~ns}$ (purple). The pump fluence at $520 \mathrm{~nm}$ is $5.5 \mu \mathrm{J} / \mathrm{cm}^{2}$ for the visible and $7.0 \mu \mathrm{J} / \mathrm{cm}^{2}$ for the near infrared measurements.

electrodes) in a time range from 3 ps to 3 ns. In Fig. 6(a), the spectra provide clear evidence of charge generation and the amplitude of the charge-induced absorption signal is stronger than that of the sample without Li additive (see Fig. S5 in the supplementary material). Most of the signal persists beyond $3 \mathrm{~ns}$, which is not observed in the sample without $\mathrm{Li}$-ions. This can be explained in terms of an increased charge generation efficiency due to fast injection aided by the presence of Li-ions and decreased back electron transfer. The spectrum at later times in panel (a) demonstrates the presence of dye cations as well as the recombination of the dye cation with electrons in $\mathrm{TiO}_{2}$ nanoparticles because the strong peak between 650 and $750 \mathrm{~nm}$ is similar to the TA spectrum of the sample without Li-ions. These peaks red-shift due to the Stark effect that interferes with the GSB of the dye molecules after insertion of Li-ions as previously reported by us. ${ }^{22,24}$ Upon addition of Li-ions, the TA spectra of $\mathbf{2}$ exhibit two pronounced absorption features as presented in panel (c): a strong absorption around $780 \mathrm{~nm}$ and an additional absorption in the NIR spectral region. These two features are assigned to the generation of dye cations created by electron injection itself facilitated by the presence of Li-ions since these features are not observed in the sample without Li addition (see Fig. S6 in the supplementary material). This was confirmed by quasisteady-state PIA measurements (Fig. 5) on the same sample showing a similar PIA spectrum. Therefore, injection appears to take place ultrafast and exciton-induced absorption is effectively absent due to the ultrafast electron injection. Clearly, the ps-ns spectra displayed in panel (c) are very similar to the spectra observed in the quasi-steady-state PIA measurement. Interestingly, the signals are fairly long-lived and even extend beyond 3-4 ns, which indicates facilitated injection in samples of dye $\mathbf{2}$ after addition of Li-ions, as already deduced from the transient PL measurements shown in Fig. 3, which demonstrated a significant change in PL lifetimes upon addition of $\mathrm{Li}$-ions. In panel (e), the positive GSB signals extend to 620 and $650 \mathrm{~nm}$, depending on the delay time chosen, and the negative PA extends up to $1500 \mathrm{~nm}$. The spectral shape of the broad negative PA signals, especially in the NIR, given in panel (e) is fairly similar to that of dye $\mathbf{3}$ on $\mathrm{TiO}_{2}$ without Li-ions (see Fig. S7.1 in the supplementary material), indicating once more that dye $\mathbf{3}$ is capable of injecting electrons even without addition of Li-ions. Furthermore, the assignment of the long-lived signals of dye $\mathbf{3}$ on $\mathrm{TiO}_{2}$ to charges is confirmed by the fluence dependence of long-time delay measurements provided in Fig. S7.2 in the supplementary material. A complete-device-like sample shown in panel (f) exhibits TA spectra in the visible similar to the spectra shown in panel (e), which is a consequence of the inefficient injection process. In addition, we note that the amplitude of the signal at long delay times in the NIR is not significantly enhanced compared to the amplitude of the signal in the visible, indicating less charge generation in dye $\mathbf{3}$ in line with the results of the IE determination presented above. It is difficult to determine if there is charge generation present on the sub-ns time domain in this sample; however, the decay in the ns- $\mu$ s measurements provides evidence for generation of charges in dye $\mathbf{3}$ on $\mathrm{TiO}_{2}$ after addition of $\mathrm{Li}$-ions.

In order to compare the dye cation kinetics, the TA signal in the wavelength region between 850 and $900 \mathrm{~nm}$ was chosen, as this spectral region is not significantly affected by the Stark effect that is present across the entire visible range. Figure 7(a) shows the ps- $\mu$ s TA kinetics of dye cations in the three samples treated with Li-ions. Interestingly, $\mathbf{3}$ exhibited a fast signal decay, while $\mathbf{1}$ and $\mathbf{2}$ exhibited a signal rise up to 100-300 ps. The observation of a short-lived signal in $\mathbf{3}$ and the low IEs obtained from our measurements shown above indicate that $\mathbf{3}$ suffers from poor charge injection. In fact, the fast decay can be interpreted as recombination of the dyes' excited-states, here the dye excitons, prior to injection. For $\mathbf{1}$ and $\mathbf{2}$, the slower decay dynamics imply increased charge generation and decreased electron-hole recombination. This is attributed to fast electron injection aided by the presence of Li-ions, leading to rising signals due to the injection from the dye excitons and concomitant generation of dye cations.

The TA measurements on device-like structures using dye 1 presented in Fig. 6(b) exhibit four interesting features: (i) broad PA spectra extending further into the high-energy part of the visible region of the probe spectrum, (ii) steadily decreasing PA at $900 \mathrm{~nm}$, (iii) a clear isosbestic point at $1350 \mathrm{~nm}$, and (iv) increasing PA beyond $1350 \mathrm{~nm}$. First, the broader PA in the visible spectral range is caused by the presence of Liions, which increase the local polarity in the vicinity of the dye molecules and lead to a shift of the excited state spectra linked to the dipole moment of the excited-state. ${ }^{22,24}$ In panel (a), the direction of the excited state dipole moment is the same as the local electric field created by the Li-ions, thereby leading to a lowering of the energy levels of the dye. Second, the signal around $900 \mathrm{~nm}$ where the absorption of dye cations is observed, decays steadily. Hence, efficient regeneration of the dye molecules' ground states seems to occur in the presence of the hole transport material spiro-OMeTAD, in turn reducing the dye cation absorption. Third, an isosbestic point at $1350 \mathrm{~nm}$ points to hole transfer from the dye cation to the spiro-OMeTAD molecules as the exclusive process observed on this time scale. Finally, the increasing signal amplitude beyond $1350 \mathrm{~nm}$ is clear evidence that dye regeneration 

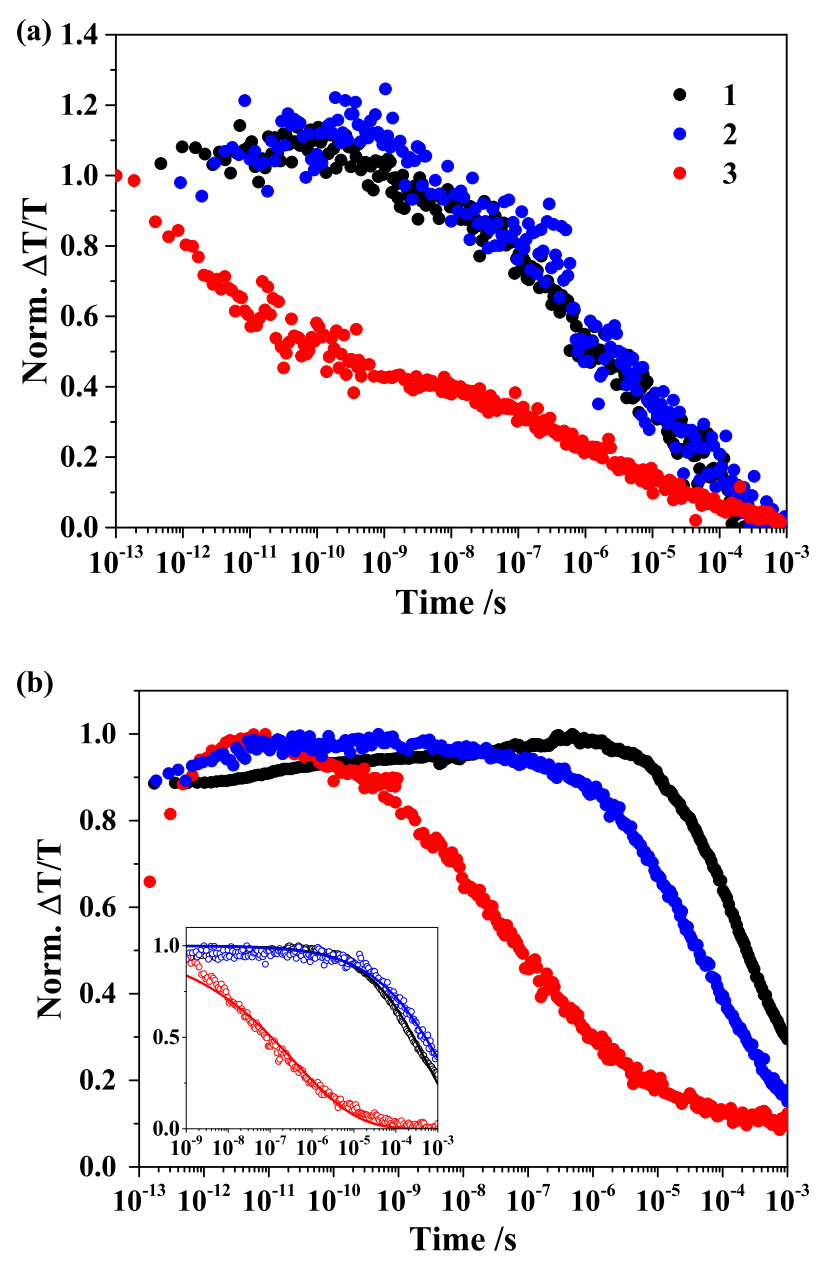

FIG. 7. Transient absorption kinetics of (a) dye cations measured on samples of the dyes adsorbed on $\mathrm{TiO}_{2}$ after addition of $\mathrm{Li}$-ions and of (b) spiro-cations in the solar cell-like samples. 1, 2, and 3 are represented by black, blue, and red lines, respectively. The inset shows the fitted dynamics.

continues beyond 3 ns. For $\mathbf{2}$, the early time absorption feature of the dye cations peaking at $780 \mathrm{~nm}$ in a complete device-like sample is very similar to that shown in panel (c) but disappears as the delay time increases due to efficient regeneration by the hole conductor, as obvious from panel (d). Strong absorption signals steadily increasing up to 3-4 ns in the NIR are a signature of the oxidation of the hole conductor due to hole transfer from the dye cations, rather than absorption of the dye cation itself. Unlike for $\mathbf{1}$, an isosbestic point is not observed here because the NIR absorption of dye cations is fairly similar to that of spiro-cations in the NIR. However, the amplitude of the signal at ns delay times (purple, $3 \mathrm{~ns}$ ) in the visible (dye cation) and NIR (hole conductor) indicates efficient hole transfer in devices using dye $\mathbf{2}$. It is worth noting the changes in amplitude of the absorption spectra of the dye $\mathbf{2}$ cations. The amplitude of the absorption spectra of the dye cations increases to 2.13 at $775 \mathrm{~nm}$ in panel (c) from 1.18 at $720 \mathrm{~nm}$ in the sample without any additive (see Fig. S6 in the supplementary material) and decreases to 1.79 at $775 \mathrm{~nm}$ due to the regeneration of dye cations in panel (d). Changes in amplitude can point to charge generation facilitated by the presence of Li-ions and to regeneration by the hole conductor. The solar cell-like sample that uses dye $\mathbf{3}$ shown in panel (f) exhibits TA spectra in the visible, which are similar to the spectra provided in panel (e) due to an inefficient electron injection process. In addition, we note that the amplitude of the signal in the NIR at later times is not significantly enhanced compared to the amplitude of the signal in the visible region. This indicates that regeneration in $\mathbf{3}$ is not as efficient as in $\mathbf{1}$ or $\mathbf{2}$.

Figure 7(b) shows the ns-ms dynamics of the spiro-cation of the three device-like samples. In these samples, $\mathbf{1}$ and $\mathbf{2}$ exhibit a slightly increasing or almost constant signal up to 1 ns-1 $1 \mu \mathrm{s}$, respectively, due to the regeneration of oxidized dye molecules, whereas 3 revealed a gradual signal decay, after an initial rise from 10 to $100 \mathrm{ps}$, due to fast recombination with electrons in titania. A gamma function was used here to fit the dynamics, and the lifetime of spiro-OMeTAD cations was determined to be $2.6 \mathrm{~ms}$ in $2,0.9 \mathrm{~ms}$ in $\mathbf{1}$, and $2.0 \mu \mathrm{s}$ in $\mathbf{3}$ indicating a significantly faster decay in dye $\mathbf{3}$. This shows that the regeneration process in $\mathbf{3}$ is short, and recombination is significantly faster in this system effectively limiting the device performance. ${ }^{25,26}$

In summary, $\mathbf{1}$ and $\mathbf{2}$ reveal similar optoelectronic properties, electron injection efficiencies, and lifetimes of separated charges. PL measurements show high IEs approaching unity and long-lived charge-separated states in device-like structures. The introduction of a planar donor building block in photosensitizers is generally seen to increase photocurrents in devices, and indeed the planar-structured $\mathbf{1}$ and $\mathbf{2}$ exhibited higher photocurrents than the dendrimer-like structure 3. ${ }^{20,27-30}$ In fact, 3 exhibited different photophysical characteristics, which appear to be less favorable for charge generation, although the sensitizer contains an electron-rich donor unit. Although a dendrimer-like starburst TPA molecule was reported to exhibit higher photovoltage compared to a sensitizer with a single TPA unit, the dye $\mathbf{3}$ investigated in this study gave rather low photovoltages and photocurrents in devices as a consequence of a low IE, in turn leading to only a small amount of charge generation. ${ }^{31,32}$ An even lower IE is observed after Li-salt treatment, as it creates a more polar local environment in the vicinity of the dye molecules, which in turn leads to even lesser electron injection.

Overall, devices incorporating dyes $\mathbf{1}$ and $\mathbf{2}$ exhibited higher PCEs than 3, but their efficiencies are rather low when compared to state-of-the-art devices reported earlier, despite the sub-ms and ms lifetime of the oxidized hole-conductor in 1-based and 2-based devices (several orders of magnitude slower than the ordinary regeneration process) and the long dye cation generation period (up to $1 \mathrm{~ns}$ ). ${ }^{15,33}$ Consequently, only $30 \%$ of the absorbed photons contribute to photocurrents in the device as deduced from the IQE measurements, whereas the photosensitizer exhibited more than $60 \%$ of the literature EQE value. ${ }^{4,17}$

\section{EXPERIMENTAL}

\section{A. Device fabrication}

FTO-coated glass substrates (Pilkington, TEC-15) were cleaned with detergent (Hellmanex III, Hellma Analytics), ethanol or 2-propanol, and acetone, for $15 \mathrm{~min}$ each and finally cleaned by argon plasma treatment. Spray pyrolysis was used to create a compact titania layer from a solution of the titania precursor diisopropoxytitanium bis(acetylacetonate) 
(Sigma-Aldrich) and ethanol in a ratio of 1:10 vol. \%. The total thickness of this compact layer was measured to be $80-100 \mathrm{~nm}$. A $2 \mu$ m-thick mesoporous $\mathrm{TiO}_{2}$ layer was deposited on top by using the doctor blade coating method and a commercial titania paste (Dyesol, DSL-18NR-T). Different thicknesses of the titania layer in devices were tested, and a thickness of $2 \mu \mathrm{m}$ was found to be the optimal. $40 \mu \mathrm{L}$ of the diluted paste was used per each film. The samples were then sintered at $500{ }^{\circ} \mathrm{C}$ for $30 \mathrm{~min}$ applying a gradual increase of the temperature. The substrates were then heated to $70{ }^{\circ} \mathrm{C}$ and treated with a titanium (IV) chloride (Sigma-Aldrich ReagentPlus ${ }^{\circledR}$, 99.9\%) solution and left for $1 \mathrm{~h}$. After the $\mathrm{TiCl}_{4}$ treatment, the substrates were rinsed with distilled water and then further sintered at $500{ }^{\circ} \mathrm{C}$ for $45 \mathrm{~min}$. The samples were then dipped in the $0.3 \mathrm{mM}$ dye solution for sensitization for $2 \mathrm{~h}$. A $121.9 \mathrm{mM}$ spiro-OMeTAD solution in chlorobenzene, $97 \mathrm{mM}$ $t$ BP (4-tert-butylpyridine, Aldrich), and $18.2 \mathrm{mM}$ Li-TFSI solution (lithium bis (trifluoromethanesulphonyl) imide salt, Aldrich) were used for deposition of the hole transport layer. The solution was spin-cast at a speed of $1000 \mathrm{rpm}$ for $5 \mathrm{~s}$ followed by a speed of $1500 \mathrm{rpm}$ for $30 \mathrm{~s}$. A silver counter electrode was thermally evaporated (Edwards FL400, AUTO 306 CRYO) at a rate below $2.5 \AA / \mathrm{s}: 1 \AA / \mathrm{s}$ for the first $100 \mathrm{~nm}$ thickness and $2 \AA / \mathrm{s}$ for the next $100 \mathrm{~nm}$, to yield a total thickness of $200 \mathrm{~nm}$. For spectroscopy, the samples using titania nanoparticles were prepared using the same procedure as for the general device preparation described above, except that not electrodes were evaporated.

\section{B. J-V characteristics}

The power conversion efficiency of the solar cell devices was measured using an AM 1.5G solar simulator (LOT-Oriel, LSE 340/850.27B) with an incident illumination power density of $100 \mathrm{~mW} / \mathrm{cm}^{2}$. In our system, one solar cell device consisted of 6 pixels with an active area of $0.1403 \mathrm{~cm}^{2}$ each and the performance of the devices was obtained by taking the average value of all 6 pixels.

\section{Quasi-steady-state photoinduced absorption spectroscopy}

Quasi-steady-state photoinduced absorption (PIA) spectroscopy was performed in a pump-probe setup consisting of a $100 \mathrm{~W}$ tungsten-halogen lamp with a LOT-Oriel Omni- $\lambda$ 300 monochromator providing the probe light and a Newport LED (LED-527-HP) emitting at $527 \mathrm{~nm}$ with a power density of ca. $100 \mathrm{~mW} \mathrm{~cm}{ }^{-2}$ at the sample position used as the pump. The transmitted light was dispersed by a second (identical) monochromator and then detected by a photodetector. For measurements in the wavelength range from 550 to $1000 \mathrm{~nm}$, an amplified silicon photodetector (Thorlabs PDA 100A) was employed, which was replaced by an amplified germanium detector (Thorlabs PDA 50B) for the wavelength range from 900 to $1800 \mathrm{~nm}$. For the IR spectral region (1700$4000 \mathrm{~nm}$ ), an amplified nitrogen-cooled InSb detector (Teledyne Judson J10D) was used. The pump light was chopped at $317 \mathrm{~Hz}$ to induce changes in the transmission $\Delta \mathrm{T}$, which were measured by using a lock-in amplifier (EG\&G Princeton Applied Research model 5210). Where necessary, the $\Delta \mathrm{T}$ signal was corrected for photoluminescence and scattered pump.
To calculate $\Delta \mathrm{T} / \mathrm{T}$, the transmission was recorded prior to the PIA measurement. The sample was kept in air during the measurements.

\section{Absorption and photoluminescence}

Absorption spectra were recorded in transmission with a Perkin Elmer Lambda 25 spectrophotometer. Photoluminescence was measured with a Hamamatsu streak camera system using a femtosecond laser (Coherent MIRA Duo) as the excitation source. The $800 \mathrm{~nm}$ output of the oscillator was doubled in a BBO crystal to get $400 \mathrm{~nm}$ excitation. The dye cation absorption was recorded in solution by chemically oxidizing the dye with iron(III)chloride. The dye anion spectrum was recorded by blending the dye into LupasolTM (solvent: DCM) and drop casting the blend on a quartz substrate followed by UV illumination. Several absorption spectra were recorded with increasing illumination time, until no further change of the absorption could be observed.

\section{E. Ultrafast transient absorption measurement}

Transient absorption measurements were performed with a home-built pump-probe setup. To measure a time range up to $4 \mathrm{~ns}$ with a resolution of $\sim 100 \mathrm{fs}$, the output of a commercial titanium:sapphire amplifier (Coherent LIBRA HE, $3.5 \mathrm{~mJ}$, $1 \mathrm{kHz}, 100 \mathrm{fs}$ ) was split with one portion used to generate a 530-550 nm excitation pulse using a home-built narrowband noncollinear optical parametric amplifier (NOPA) and another used to generate a $1300 \mathrm{~nm}$ seed pulse [output of an optical parametric amplifier (Coherent OPerA Solo)] for white-light generation in the visible to NIR (500-1000 nm) in a c-cut $3 \mathrm{~mm}$ thick sapphire window. For the redder NIR white-light (1200-2000 nm), a $2100 \mathrm{~nm}$ seed was used. A polished silicon substrate was added into the path of the white light to block the visible fraction of the supercontinuum. The variable delay of up to $4 \mathrm{~ns}$ between the pump and probe was introduced by a broadband retroreflector mounted on a mechanical delay stage. The excitation pulse was chopped at $500 \mathrm{~Hz}$, while the white-light pulses were dispersed onto a linear silicon (Hamamatsu NMOS linear image sensor S3901) or Peltier-cooled extended InGaAs (Entwicklungsbüro Stresing) photodiode array for the VIS and NIR light, respectively. The arrays were read out at $1 \mathrm{kHz}$. Adjacent diode readings corresponding to the transmission of the sample after an excitation pulse and without an excitation pulse were used to calculate $\Delta \mathrm{T} / \mathrm{T}$. Samples were excited with several different fluences in the range of $3-20 \mu \mathrm{J} / \mathrm{cm}^{2}$. For measuring in the time range of $1 \mathrm{~ns}-1 \mathrm{~ms}$ with a resolution of $600 \mathrm{ps}$, the excitation pulse was provided by an actively Q-switched Nd:YVO4 laser (AOT Ltd., MOPA) at $532 \mathrm{~nm}$. The delay between the pump and probe in this case was controlled by an electronic delay generator (Stanford Research Systems DG535). TA measurements were performed at room temperature under dynamic vacuum at pressures.

\section{CONCLUSIONS}

In this study, we determined the photophysical processes that limit the performance of a series of novel donor- $\pi$ bridgeacceptor photosensitizers in solid-state dye-sensitized solar 
cells. Dyes 1 and 2 allowed efficient electron injection, fast dye regeneration, and slow carrier recombination, which are all beneficial for device performance. We also believe that the flattened structure of dyes $\mathbf{1}$ and $\mathbf{2}$ leads to more efficient charge transport. Furthermore, electron injection can be further enhanced in the presence of $\mathrm{Li}$-ions, which is beneficial for device performance. On the other hand, dye structure 3 gave the less efficient electron injection, fast interfacial electron-dye cation recombination, slower dye regeneration by the HTM spiro-MeOTAD, and also faster charge recombination, which all in all limit the device performance. Overall dye structures 1 and 2 appear to be more promising for further device efficiency optimization and as structural motifs for future material development.

\section{SUPPLEMENTARY MATERIAL}

See supplementary material for detailed information about the synthesis of the dyes, DFT calculations, electrochemical, and spectroscopic data.

\section{ACKNOWLEDGMENTS}

F. Laquai thanks the Max Planck Society for funding the Max Planck Research Group.

${ }^{1}$ B. O'Regan and M. Grätzel, Nature 353, 737 (1991).

${ }^{2}$ Y. Bai, J. Zhang, D. Zhou, Y. Wang, M. Zhang, and P. Wang, J. Am. Chem. Soc. 133, 11442 (2011).

${ }^{3}$ J. Liu, J. Zhang, M. Xu, D. Zhou, X. Jing, and P. Wang, Energy Environ. Sci. 4, 3021 (2011).

${ }^{4}$ A. Yella, H. Lee, H. N. Tsao, C. Yi, A. K. Chandiran, M. Nazeeruddin, E. W. Diau, C. Yeh, S. M. Zakeeruddin, and M. Grätzel, Science 334, 629 (2011); J. Burschka, A. Dualeh, F. Kessler, E. Baranoff, N. Cevey-Ha, C. Yi, M. K. Nazeeruddin, and M. Grätzel, J. Am. Chem. Soc. 133, 18042 (2011).

${ }^{5}$ M. K. Nazeeruddin, A. Kay, I. Rodicio, R. Humphry-Baker, E. Mueller, P. Liska, N. Vlachopoulos, and M. Graetzel, J. Am. Chem. Soc. 115, 6382 (1993).

${ }^{6}$ M. K. Nazeeruddin, P. Péchy, T. Renouard, S. M. Zakeeruddin, R. HumphryBaker, P. Comte, P. Liska, L. Cevey, E. Costa, V. Shklover, L. Spiccia, G. B. Deacon, C. A. Bignozzi, and M. Grätzel, J. Am. Chem. Soc. 123, $1613(2001)$

${ }^{7}$ P. Wang, S. M. Zakeeruddin, J. E. Moser, M. K. Nazeeruddin, T. Sekiguchi, and M. Grätzel, Nat. Mater. 2, 402 (2003).

${ }^{8}$ M. K. Nazeeruddin, F. De Angelis, S. Fantacci, A. Selloni, G. Viscardi, P. Liska, S. Ito, B. Takeru, and M. Grätzel, J. Am. Chem. Soc. 127, 16835 (2005).

${ }^{9}$ A. Yella, R. Humphry-Baker, B. F. E. Curchod, N. A. Astani, J. Teuscher, L. E. Polander, S. Mathew, J.-E. Moser, I. Tavernelli, U. Rothlisberger, and M. Grätzel, Chem. Mater. 25(13), 2733 (2013).
${ }^{10}$ J. Yum, T. W. Holcombe, Y. Kim, K. Rakstys, T. Moehl, J. Teuscher, J. H. Delcamp, M. K. Nazeeruddin, and M. Grätzel, Sci. Rep. 3, 2446 (2013).

${ }^{11}$ S. Kim, J. Lee, S. Kang, J. Ko, J.-H. Yum, S. Fantacci, F. De Angelis, D. Di Censo, Md. K. Nazeeruddin, and M. Grätzel, J. Am. Chem. Soc. 128(51), 16701 (2006).

${ }^{12}$ B. Chen, D. Chen, C. Chen, C. Hsu, H. Hsu, K. Wu, S. Liu, P. Chou, and Y. Chi, J. Mater. Chem. 21, 1937 (2011).

${ }^{13}$ M. Planells, L. Pellejà, J. N. Clifford, M. Pastore, F. De Angelis, N. López, S. R. Marder, and E. Palomares, Energy Environ. Sci. 4, 1820 (2011).

${ }^{14}$ D. Zhou, Q. Yu, N. Cai, Y. Bai, Y. Wanga, and P. Wang, Energy Environ. Sci. 4, 2030 (2011).

${ }^{15}$ Y. Cao, N. Cai, Y. Wang, R. Li, Y. Yuan, and P. Wang, Phys. Chem. Chem. Phys. 14, 8282 (2012).

${ }^{16}$ N. Cai, Y. Wang, M. Xu, Y. Fan, R. Li, M. Zhang, and P. Wang, Adv. Funct. Mater. 23(14), 1846 (2013).

${ }^{17}$ J. Yum, E. Baranoff, F. Kessler, T. Moehl, S. Ahmad, T. Bessho, A. Marchioro, E. Ghadiri, J.-E. Moser, C. Yi, Md. K. Nazeeruddin, and M. Grätzel, Nat. Commun. 3, 631 (2012).

${ }^{18}$ H. Cheng, K. Y. Chiu, S. H. Lu, C. Chen, Y. W. Lee, T. Yang, M. Y. Kuo, P. P. Chen, and Y. O. Su, J. Phys. Chem. A 119(10), 1933 (2015).

${ }^{19}$ L. Cai, H. N. Tsao, W. Zhang, L. Wang, Z. Xue, M. Grätzel, and B. Liu, Adv. Energy Mater. 3(2), 200 (2013).

${ }^{20}$ H. N. Tsao, C. Yi, T. Moehl, J. Yum, S. M. Zakeeruddin, M. K. Nazeeruddin, and M. Grätzel, ChemSusChem 4(5), 591 (2011).

${ }^{21}$ I. A. Howard, M. Meister, B. Baumeier, H. Wonneberger, N. Pschirer, R. Sens, I. Bruder, C. Li, K. Müllen, D. Andrienko, and F. Laquai, Adv. Energy Mater. 4(2), 1300640 (2014).

${ }^{22}$ M. Fakis, M. Dori, E. Stathatos, H.-H. Chou, Y.-S. Yen, J. T. Lin, V. Giannetas, and P. Persephonis, J. Photochem. Photobiol., A 251, 18 (2013).

${ }^{23}$ M. Meister, B. Baumeier, N. Pschirer, R. Sens, I. Bruder, F. Laquai, D. Andrienko, and I. A. Howard, J. Phys. Chem. C 117(18), 9171 (2013).

${ }^{24}$ U. B. Cappel, A. L. Smeigh, S. Plogmaker, E. M. J. Johansson, H. Rensmo, L. Hammarström, A. Hagfeldt, and G. Boschloo, J. Phys. Chem. C 115(10), 4345 (2011).

${ }^{25}$ M. Xu, D. Zhou, N. Cai, J. Liu, R. Li, and P. Wang, Energy Environ. Sci. 4, 4735 (2011).

${ }^{26}$ A. Y. Anderson, P. R. F. Barnes, J. R. Durrant, and B. C. O'Regan, J. Phys. Chem. C 115(5), 2439 (2011).

${ }^{27}$ S. I. Wharton, J. B. Henry, H. McNab, and A. R. Mount, Chem. - Eur. J. 15, 5482 (2009).

${ }^{28}$ D. P. Hagberg, T. Edvinsson, T. Marinado, G. Boschloo, A. Hagfeldt, and L. Sun, Chem. Commun. 0, 2245 (2006).

${ }^{29}$ A. W. Jones, M. Louillat-Habermeyer, and F. W. Patureau, Adv. Synth. Catal. 357(5), 945 (2015).

${ }^{30}$ P. Kautny, D. Lumpi, Y. Wang, A. Tissot, J. Bintinger, E. Horkel, B. Stöger, C. Hametner, H. Hagemann, D. Mab, and J. Fröhlich, J. Mater. Chem. C 2, 2069 (2014).

${ }^{31}$ Z. Ning, Q. Zhang, W. Wu, H. Pei, B. Liu, and H. Tian, J. Org. Chem. 73(10), 3791 (2008).

${ }^{32}$ R. Li, J. Liu, N. Cai, M. Zhang, and P. Wang, J. Phys. Chem. B 114(13), 4461 (2010).

${ }^{33}$ U. Bach, Y. Tachibana, J.-E. Moser, S. A. Haque, J. R. Durrant, M. Grätzel, and D. R. Klug, J. Am. Chem. Soc. 121(32), 7445 (1999). 